Hanna Kaczmarek

Jan-Długosz-Akademie, Częstochowa

DOI: $10.19195 / 0435-5865.142 .15$

\title{
Similaritäts- und Kontrastrelationen in einem Lernerwörterbuch. Lemmatisierungsschwierigkeiten
}

\section{Zum Ziel des Beitrags}

Bei sprachproduktiven und sprachrezeptiven Aufgaben konsultieren Fremdsprachler häufig ein einsprachiges Wörterbuch, in der Hoffnung, passende, situationsangemessene Wendungen, synonyme oder antonyme Wörter zu finden, um ihren Text einerseits inhaltlich aussagekräftiger, andererseits stilistisch abwechslungsreicher zu gestalten. Das Wörterbuch scheint für den fremdsprachigen Benutzer die beste verfügbare Informationsquelle zu sein, die ihm die möglichen Bedeutungs- und Formulierungsfehler zu vermeiden hilft.

Das Anliegen dieses Beitrags ist es, aufgrund der kontrastierenden Analyse der gewählten Wörterbuchartikel aufzuzeigen, welche Lexeme in den gängigen deutschen Lernerwörterbüchern ${ }^{1}$ als Synonyme bzw. Antonyme fungieren und wie diese lemmatisiert werden. Im Folgenden soll untersucht werden, inwieweit die bei den paradigmatischen Austauschklassen stehenden Informationen dem potentiellen fremdsprachigen Wörterbuchbenutzer bei möglichen Fragestellungen weiterhelfen können.

\section{Zu lexikographischen Prinzipien der Lernerwörterbücher}

In der Metalexikographie wird neben dem Terminus Lernerwörterbuch die Bezeichnung Lernwörterbuch verwendet. Der größte Unterschied zwischen den beiden Wörterbuchtypen - so Bergenholtz (2002: 39) - besteht in ihren unterschied-

${ }^{1}$ Untersucht werden folgende einsprachige Lernerwörterbücher: Langenscheidt. Großwörterbuch Deutsch als Fremdsprache (2003) (= LGWDaF), Pons. Großwörterbuch Deutsch als Fremdsprache (2004) (= PGWDaF), Wahrig. Großwörterbuch Deutsch als Fremdsprache (2008) (=WGWDaF) und de Gruyter. Wörterbuch Deutsch als Fremdsprache (2000) (= dGWDaF). 
lichen Funktionen. Während das Lernwörterbuch prinzipiell als Instrument für die Erweiterung und Vertiefung der Sprachkenntnisse eines Lerners konzipiert ist, und somit die Lernfunktion erfüllt, wird das Lernerwörterbuch primär bei der Rezeption und Produktion von Texten genutzt. Beide Wörterbuchtypen verfolgen demnach verschiedene Ziele: Lernerwörterbücher werden hauptsächlich zum punktuellen Nachschlagen unbekannter Wörter benutzt, die Lernwörterbücher dienen hingegen der „kontrollierten Kompetenzerweiterung“ (Zöfgen 1994: 17). Bei einem Lernwörterbuch liegt der Fokus auf der für das Vokabellernen geeignetsten Darstellung eines begrenzten Wortschatzausschnitts, der vom Lerner aktiv beherrscht werden soll (vgl. Runte 2015: 127).

Die gängigen DaF-Wörterbücher, ${ }^{2}$ die stark auf die Bedürfnisse der Benutzer gerichtet sind, weichen jedoch in ihrer Konzeption von den Grundprinzipien der beiden Wörterbuchtypen so ab, dass sie nicht als reine Vertreter der oben genannten Wörterbuchtypen einzustufen sind. Sie stellen eher eine Mischform dar, indem sie wissens- und textbezogene Funktionen zu verbinden versuchen. Daher wird in diesem Beitrag zwischen den beiden Wörterbuchtypen nicht unterschieden und beide Termini werden synonym verwendet.

Die spezifische Struktur der Lernerwörterbücher, die an den fremdsprachigen Benutzer adressiert sind, ergibt sich daraus, dass sie versuchen, „als selektive Wörterbücher in ihrer Lemmaauswahl und Präsentation auf dessen spezifische Bedürfnisse einzugehen“ (Töpel 2011: 25). Dies kommt deutlich in dem besonderen relationalen Verhältnis von Makro- und Mikrostruktur zum Ausdruck. Der umfangreichen Mikrostruktur, die die detaillierten Informationen zur Grammatik, Bedeutung des Lemmas, Kollokation, Idiomatik und Aussprache angibt, wird die selektive Makrostruktur entgegengesetzt. In derartigen Nachschlagewerken liegt der Schwerpunkt demnach nicht so sehr in der Quantität der Stichwörter, sondern in der Qualität des lexikographischen Eintrags. Das bedeutet, dass die Bedeutungsbeschreibung der Lemmata möglichst einfach und verständlich präsentiert werden soll und die angegebenen Bedeutungen oder Bedeutungsvarianten sich klar von denen der ähnlichen Lexeme abheben müssen (vgl. Wellmann 2004: 91). Eine besondere Rolle spielen daher die vorgeschlagenen Beispiele, die die Kontextualisierung des Lemmas erfolgreich zu erschließen helfen. Die Anzahl der im Wörterbuch verzeichneten Lemmata ist dabei zweitrangig.

Zur Verdeutlichung der Stichwortbedeutung dienen außer der klassischen Bedeutungsparaphrasen zusätzliche ergänzende Angaben, zu denen insbesondere Synonyme und Antonyme gehören. Zusammen mit Kollokationen und Anwendungsbeispielen bilden sie das lexikalische Umfeld des Lemmas, wodurch semantische Vernetzungen und relationale Beziehungen zwischen den einzelnen Lexemen angedeutet werden können. Diese Art der integrativen Bedeutungserläuterung, die als zweckmäßig zu bewerten ist, weist dem Lernenden den Weg von

${ }^{2}$ Im Deutschen werden die für didaktische Zwecke konzipierten Wörterbücher Wörterbücher Deutsch als Fremdsprache (DaF-Wörterbücher oder L2-Wörterbücher) genannt. 
einem Stichwort zu den lexikalischen Alternativen (aufgrund der Similaritäts- und Kontrastrelationen), so dass Wörter in einer Äußerungskette in derselben Position ausgetauscht werden können.

\section{Similaritätsrelationen in einem Lernerwörterbuch}

Synonyme nehmen in der Regel die Position nach der Bedeutungsparaphrase ein, ${ }^{3}$ wobei ,pro Bedeutung des Lemmazeichens [...] höchstens drei Wortsynonyme genannt werden dürfen“ (Wiegand 2013: 21). Sie erfüllen dabei drei verschiedene Funktionen: sie sind eine Art der Zusammenfassung der Definition (1), sie ergänzen die Bedeutung (2) (vgl. LGWDaF 2003: XVIII) oder sie ersetzen die Definition (3):

(1) Irre, der/die; -n, -n; [...] j-d, der psychisch od. geistig krank ist $\approx$ Geisteskranke(r), Verrückte(r), Wahnsinnige(r) (LGWDaF 2003: 544)

Egoismus, der $\sim,<o . P l$. $>$, nur auf den eigenen Vorteil bedachtes Verhalten, Handeln'; SYN Selbstsucht (dGWDaF 2000: 248)

(2) Galle, die; -, - $n 2$ ein (Körper)Organ, in dem die G. (1) gespeichert wird $\approx$ Gallenblase (LGWDaF 2003: 388)

Eigentümer, der $\sim S$, ,jmd., der über ein Eigentum (1) verfügen kann und das Recht hat, es zu nutzen'; SYN Besitzer (dGWDaF 2000: 252)

(3) Eiscreme, a. Eiskrem (e) die <-, $-s>$ ( $\approx$ Speiseeis) (PGWDaF 2004: 362)

Zahncreme, $<$ die; -, $-s>=$ Zahnpasta (WGWDaF 2008: 1175)

Die angeführten Beispiele sind ein Beweis dafür, dass die Erscheinung der Synonymie in der Lernerlexikographie äußerst problematisch ist: ,statt von Bedeutungsgleichheit zu sprechen, wird mehrheitlich von Bedeutungsähnlichkeit als der eigentlichen Synonymie ausgegangen" (Wanzeck 2010: 86). Dies ist insoweit verständlich, als in der Sprache kaum Lexeme vorkommen, deren denotative und konnotative Bedeutungen sich vollkommen decken würden. „Eine vollständige, 100\%ige Bedeutungsidentität liegt kaum vor, also eine totale, absolute oder reine Synonymie ist unwahrscheinlich, weil sich eine Sprachgemeinschaft niemals verschiedene Formen mit übereinstimmenden Bedeutungen leisten würde“ (Knipf-Komlosi, Rada, Bernath 2006: 158). Solche Lexempaare wie Sonnabend - Samstag, ${ }^{4}$ anfangen beginnen oder Aprikosen - Marillen werden zwar als Beispiele für totale Synonymie angeführt, in der Tat handelt es sich jedoch um territoriale Dubletten, die sich in der Standardsprache als bedeutungsgleichwertige Pendants eingebürgert haben.

${ }^{3}$ In PGWDaF stehen die Angaben zur Synonymie und Antonymie direkt nach dem Stichwort und vor der Bedeutungsparaphrase. In WGWDaF werden Synonyme, abhängig davon, ob sie für mehrere Bedeutungen gültig sind, oder ob sie nur einzelne Bedeutungsvarianten betreffen, entweder vor der Bedeutungserklärung oder unmittelbar danach aufgeführt.

${ }^{4} \mathrm{Zu}$ Kontroversen über das Wortpaar Sonnabend - Samstag (vgl. u.a. Knipf-Komlosi, Rada, Bernath 2006: 158; Wanzeck 2010: 87). 
Demnach werden in den analysierten Lernerwörterbüchern recht selten Lexeme verzeichnet, die aufgrund eines identischen begrifflichen Kerns in gleicher syntaktischer Umgebung und in den gemeinsamen Kontextverbindungen vorkommen können. Viel häufiger werden als Synonyme solche Lexeme betrachtet, die entweder verschiedenen Stilebenen zuzurechnen sind (Gesicht vs. Antlitz), oder solche Wortpaare, die aus einem nativen Wort und einem Fremdwort (Fahrstuhl vs. Lift) oder aus einem Fachausdruck und einem Laienwort (Zahnersatz vs. Prothese) bestehen. Auch regional gebrauchte Lexeme sind in den Lernerwörterbüchern als Synonyme gekennzeichnet (Streichholz vs. Zündholz, Fleischer vs. Metzger).

In den Erläuterungen zur Konzeption des jeweiligen Wörterbuchs wird lediglich eine ganz allgemeine Definition des Synonyms (Synonyme sind bedeutungsähnliche oder bedeutungsgleiche Wörter) angegeben, ohne dass auf ihre Spezifik genauer eingegangen wird. Nur dGWDaF nennt das Kriterium der kontextuellen Austauschbarkeit, anhand dessen Lexeme als Synonyme betrachtet werden können (vgl. dGWDaF 2000: X). Obwohl in WGWDaF auch auf die Bedeutungsgleichheit und -ähnlichkeit der synonymen Lexeme hingewiesen wird (vgl. WGWDaF 2008: 10), werden manche Lexeme als Synonyme markiert (Syn), andere dagegen, die der Definition nach auch zu Synonymen zugerechnet werden könnten, werden mit $(=)$ gekennzeichnet. Synonyme werden hier definiert als „,bedeutungsgleiche oder bedeutungsähnliche Wörter" (WGWDaF 2008: 10). In der Tabelle mit Zeichen und Abkürzungen wird dagegen erklärt, dass das Gleichheitszeichen „Hinweis auf ein Wort mit gleicher Bedeutung" (WGWDaF 2008: 13) ist. Eine ausführliche Erläuterung, wo der grundlegende Unterschied zwischen den mit (Syn) und jenen mit (=) markieren Lexemen liegt, fehlt. Es scheint, als ob mit dem Gleichheitszeichen totale Synonyme (4) gekennzeichnet worden wären:

(4) Semmel $=$ Brötchen

Kassenbon $=$ Kassenzettel

Abendbrot $=$ Abendessen

Zahnersatz $=$ Prothese

Wegen der fehlenden Erklärung, welche Lexeme als totale und welche für partielle Synonyme gehalten werden, kann nur angenommen werden, dass regionale Entsprechungen, Fremdwörter und Fachausdrücke als totale Synonyme behandelt werden. Dieses Prinzip wird aber nicht konsequent befolgt und bestimmte Wortpaare werden trotz ihrer Bedeutungsgleichheit mit (Syn) markiert (5) oder ihre Bedeutungsgleichheit bleibt überhaupt nicht bezeichnet (6):

(5) Zahnmedizin - Syn Zahnheilkunde

Grippe - Syn Influenza

Leihwagen - Syn Leihauto 
(6) vertikal-senkrecht

Fahrstuhl-Lift

Dotter - Eigelb

Wortpaare, die zwar eine oder mehrere Bedeutungsvarianten haben, aber verschiedenen Stilebenen angehören und dadurch nur kontextbedingt ausgetauscht werden können, werden weder mit dem Gleichheitszeichen noch mit der Abkürzung (Syn) markiert. Sie sind somit keine Synonyme (7):

(7) Antlitz (poet.) - Gesicht

Drahtesel (umg.; scherz.) - Fahrrad

Gatte - (geh.) Ehemann

In den übrigen gesichteten Lernerwörterbüchern (LGWDaF, PGWDaF) wird zwischen den totalen und partiellen Synonymen nicht unterschieden. Als Synonyme werden jeweils Lexeme mit ungefähr gleicher Bedeutung betrachtet.

Die Vorgehensweise des dGWDaF bei der Auswahl und Angabe der Synonyme ist dagegen viel exakter und anschaulicher. Bei Bedeutungsgleichheit wird die Bedeutungserklärung eines Lexems mithilfe eines Synonyms angegeben (8):

(8) Habsucht - Syn Habgier

Busen - Syn Brust

Gauner - Syn Betrüger

Wenn die Austauschbarkeit der Lexeme stilistisch begrenzt ist, so wird als erstes das neutrale Grundsynonym (9) angeführt, das „mit einer umschreibenden Bedeutungserklärung versehen“ ist (dGWDaF 2000: X). Dies ermöglicht dem Lerner ,vom Einzelwort zu den Bedeutungsgleichen od. -ähnlichen vorzudringen“ (dGWDaF 2000: X):

(9) Gaul-umg. emot. neg. Syn Pferd

Gesicht-Syn Antlitz, Fratze, Fresse

Das unterschiedliche, uneinheitliche lexikographische Verfahren, das in den gesichteten Lernerwörterbüchern angewendet wird, hat zur Folge, dass bestimmte Lexeme nicht immer als Synonyme klassifiziert werden, was die folgende Tabelle verdeutlicht: 
Tab. 1. Stichwörterpaare/ -gruppen in den Lernerwörterbüchern (in alphabetischer Reihenfolge)

\begin{tabular}{|c|c|c|c|c|}
\hline Stichwort & WGWDaF & LGWDaF & PGWDaF & dGWDaF \\
\hline Eigelb & Dotter & $\approx$ Dotter & $\approx$ Eidotter & SYN Dotter \\
\hline (Ei)Dotter & -5 & $\approx$ Eigelb & $\approx$ Eigelb & SYN Eigelb \\
\hline Fahrstuhl & Aufzug, Lift & $\approx$ Aufzug, Lift & $\approx$ Aufzug, Lift & SYN Lift \\
\hline Lift & $\begin{array}{l}\text { Aufzug, Fahr- } \\
\text { stuhl }\end{array}$ & $\begin{array}{l}\approx \text { Aufzug, Fahr- } \\
\text { stuhl }\end{array}$ & $\approx$ Fahrstuhl & SYN Fahrstuhl \\
\hline Gatte & geh. Ehemann & $\approx$ Ehemann & geh. Ehemann & $\begin{array}{l}\text { geh. SYN Ehe- } \\
\text { mann }\end{array}$ \\
\hline Ehemann & - & $\approx$ Mann & - & $\begin{array}{c}\text { SYN Gatte, } \\
\text { Gemahl, Mann }\end{array}$ \\
\hline Glotze & $\begin{array}{c}\text { abwertend Fern- } \\
\text { sehapparat }\end{array}$ & $\begin{array}{l}\text { gespr pej } \approx \text { Fern- } \\
\text { sehapparat }\end{array}$ & - & - \\
\hline Fernsehapparat & $=$ Fernsehgerät & $\approx$ Fernseher & - & - \\
\hline Fernsehgerät & $\begin{array}{c}\text { Syn Fernsehap- } \\
\text { parat }\end{array}$ & - & - & - \\
\hline Fernseher & $=$ Fernsehgerät & $\approx$ Fernsehapparat & - & $\begin{array}{l}\text { umg. Fernseh- } \\
\text { gerät }\end{array}$ \\
\hline Halunke & $\begin{array}{l}\text { abwertend Gau- } \\
\text { ner, Betrüger, } \\
\text { Schwindler }\end{array}$ & $\begin{array}{l}\text { oft hum } \approx \text { Schur- } \\
\text { ke, Gauner }\end{array}$ & abwert. $\approx$ Gauner & - \\
\hline Gauner & - & $\begin{array}{c}\text { gespr } \approx \\
\text { Schwindler }\end{array}$ & - & SYN Betrüger \\
\hline Schuster & $=$ Schuhmacher & $\approx$ Schuhmacher & $\approx$ Schuhmacher & - \\
\hline Schuhmacher & - & - & - & - \\
\hline Sonnabend & - & nordd $\approx$ Samstag & norddt. Samstag & SYN Samstag \\
\hline Samstag & $\begin{array}{l}\text { Syn (westfäl.; } \\
\text { ostfries.) Sater- } \\
\text { tag, } \\
\text { (bes. nord- u. } \\
\text { mitteldt.) Sonn- } \\
\text { abend }\end{array}$ & $\approx$ Sonnabend & - & SYN Sonnabend \\
\hline Unfug & $\begin{array}{l}\text { Unsinn, Dumm- } \\
\text { heiten }\end{array}$ & $\approx$ Unsinn & - & SYN Unsinn \\
\hline Unsinn & Unfug treiben & $\approx$ Blödsinn & - & $\begin{array}{l}\text { SYN Käse, Kohl, } \\
\text { Tinnef, Unfug, } \\
\text { Zimt, Zinnober }\end{array}$ \\
\hline
\end{tabular}

Die obige Zusammenstellung macht ersichtlich, dass relativ große Diskrepanzen im Hinblick auf die Synonymie von einem Wörterbuch zu einem anderen zu beobachten sind. Die Durchsicht der angeführten Lemmata lässt einige kritische

${ }^{5}$ Dieses Zeichen steht, wenn im Wörterbuchartikel kein Synonym angegeben ist. 
Hinweise formulieren, die einerseits formale, andererseits inhaltliche Behandlung der Synonyme betreffen:

1. Die Abweichungen in der Darstellungsform der Synonyme im Rahmen desselben Wörterbuchs führen dazu, dass die Bedeutungsbeziehungen, die zwischen dem Lemma und anderen Lexemen bestehen, nicht unmissverständlich verdeutlicht werden. Dieser Vorwurf trifft besonders auf WGWDaF zu, in dem bedeutungsähnliche Lexeme unreflektiert in der lexikographischen Definition verarbeitet und somit nicht als Synonyme gekennzeichnet werden (Eigelb - Dotter, Fahrstuhl-Lift, Unfug - Unsinn).

2. Die Gründe für den Verzicht auf die gesonderte Markierung der bedeutungsähnlichen Lexeme und die nicht einheitliche Markierungspraxis der Synonyme in WGWDaF (Syn oder =) müssten in den Benutzungshinweisen deutlicher erläutert bzw. im ganzen Wörterbuch vereinheitlicht werden. Die fehlenden oder unzureichenden Erklärungen können auf den Benutzer verwirrend wirken (Fernsehapparat $=$ Fernsehgerät, Fernsehgerät - Syn Fernsehapparat).

3. Unzulänglichkeiten und Defizite sind überdies bei der Angabe des betreffenden synonymen Partners festzustellen: In den Wörterbuchartikeln werden nicht immer die entsprechenden Synonyme angeführt. So z.B. steht bei Halunke $\approx$ Gauner als Synonym, aber bei Gauner würde der Lerner vergebens nach dem Synonym Halunke suchen (PGWDaF). Solche Praxis erschwert die Interpretierbarkeit der Informationen und stellt die Vollständigkeit der Angaben in Frage. Manchmal werden als Synonyme Lexeme angegeben, die von den Muttersprachlern kaum benutzt werden: z.B. Leihauto (WGWDaF).

4. Bei Wortpaaren, die aus einem standardsprachlichen und einem stilistisch gefärbten Wort bestehen, wird manchmal auf die Angabe des synonymen Partners verzichtet (Ehemann - Gatte, Fernseher - Glotze). Die in dGWDaF angewandte Vorgehensweise, die Synonymreihungen von einem stilistisch neutralen bis stilistisch markierten Wort (oder umgekehrt) aufzulisten, wird von Deutschlernern gewinnbringend als Semantisierungshilfe benutzt (Ehemann - SYN Gatte, Gemahl, Mann; Unsinn - SYN Käse, Kohl, Tinnef, Unfug, Zimt, Zinnober).

5. Bei standardsprachlichen und regionalen Differenzierungen fehlen manchmal die synonymen Entsprechungen (Samstag - Sonntag).

6. Im Falle der bedeutungsähnlichen Lexeme, die hinsichtlich der regionalen oder stilistischen Dimension verschiedenen Sprachebenen zugeordnet werden, oder deren Verwendungsmöglichkeiten stark eingeschränkt werden, sind aus lernerlexikographischer Sicht die in den Erläuterungsteil der Artikeltexte integrierten zusätzlichen Informationen zu begrüßen. Kommentare solcher Art treten z.B. unter dem Stichwort Gatte in LGWDaF und in dGWDaF auf:

Gatte-NB Für viele Sprecher sind Gatte u. Gattin veraltende Wörter. Ehepartner verwenden die Bezeichnungen meine Frau bzw. mein Mann (LGWDaF 2003: 392).

Merke: Gatte wird nicht für die Bezeichnung des eigenen Ehemannes od. des Ehemannes einer geduzten Person gebraucht (dGWDaF 2000: 374). 


\section{Kontrastrelationen in einem Lernerwörterbuch}

Zur Verdeutlichung und Veranschaulichung der bestehenden Vernetzungen zwischen den Wortbedeutungen werden in den Lernerwörterbüchern außer den Relationen der Bedeutungsgleichheit und Bedeutungsähnlichkeit die Relationen der Gegensätzlichkeit berücksichtigt. Antonyme ${ }^{6}$ Ausdrücke sollen Aufschluss über die Kontrastierungen in der Bedeutungsstruktur der einzelnen Lexeme geben, die prinzipiell als entgegengesetzte Extreme auf einer Skala anzusiedeln sind (vgl. Wiegand et al. 2010: 541). Die unter dem Oberbegriff der Antonymie erfassten Gegensatzrelationen können dabei vielfältiger Natur sein. Bereits folgende Beispiele wie tot-lebendig, gesund - krank, gut - schlecht, der Tag-die Nacht, aufmachen - zumachen lassen erkennen, dass die jeweiligen Wortpaare in unterschiedlichen oppositionellen Relationen zueinander stehen. Abzugrenzen sind voneinander - so Lyons (1980: 281-300) - Fälle der konträren, kontradiktorischen (auch komplementären), konversen und reversiven Antonymie (vgl. Schippan/ Ehrhardt 2001: 87, Wanzeck 2010: 65, Harm 2015: 76). Unter konträrer oder gradueller Antonymie (auch Antonymie im engeren Sinne) wird eine im Wesentlichen adjektivische Lexeme betreffende Kontrastrelation verstanden, „die auf einen Meß- oder Wertungsbereich mit unterschiedlichen Segmentbildungen bezogen ist" (Wanzeck 2010: 40). Möglich sind Übergangstufen, die auf einer Achse der Möglichkeiten die mittleren Positionen zwischen den Polen annehmen:

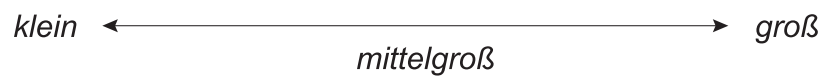

Es handelt sich also „um eine abgestufte Polarität, wobei die Antonyme die äußersten Pole einer Polaritätsachse versprachlichen" (Knipf-Komlosi / Rada / Bernath 2006: 157). Bei kontradiktorischer Antonymie gilt als Grundprinzip die Annahme, „wenn X ein a ist, ist es nicht ein b“ (Wiegand et al 2010: 540). So impliziert die Geltung des einen Lexems die Nichtgeltung des anderen: jmd., der tot ist, ist nicht lebendig, der Tag ist nicht die Nacht, tadeln ist nicht loben. Die polaren Lexeme schließen einander aus, indem sie in einer Entweder-oder - Relation zueinander stehen. Somit ist kein mittlerer Bereich zwischen den Gegensatzpolen möglich. Die konverse Gegensatzrelation besteht zwischen den zu Paaren gruppierten Lexemen, die denselben Sachverhalt aus verschiedenen Blickrichtungen perspektiveren. Die Bedeutungen der Gegenwörter beziehen sich aufeinander, indem sie einander ergänzende Rollen in demselben Prozess benennen: Lehrer Schüler, links - rechts, geben - bekommen. Gelegentlich gilt insbesondere für verbale Lexeme die reversive Relation, die im Bezug auf zeitliche Prozesse entsteht. Die Kontrastpaare bezeichnen den Anfangs- und Endpunkt eines Geschehens ( $h i$ -

\footnotetext{
${ }^{6}$ In diesem Beitrag werden Antonyme unter dem inhaltlich-begrifflichen Aspekt untersucht.
} 
naufgehen - hinuntergehen, sich anziehen - sich ausziehen, anfangen - aufhören), wobei diese Gegensatzbeziehungen umkehrbar sind.

In den analysierten Lernerwörterbüchern gelten die in die Wörterbuchartikel integrierten Gegenwörter als Semantisierungshilfe, wobei sie nur dann eingesetzt werden, ,wenn sie als Ergänzung zur Bedeutungserklärung sinnvoll erscheinen“ (WGWDaF 2008: 11). Antonyme weisen auf ,strukturelle Zusammenhänge im Wortschatz" (PGWDaF 2004: 12) hin und dienen somit gleichzeitig der Wortschatzerweiterung (vgl. LGWDaF 2003: XVIII). Im Unterschied zu den Synonymenangaben werden Gegenwörter nicht ,als selbständige Bedeutungserklärung verwendet, da der Umweg über den Gegensatz dem Benutzer nur bedingt bei der Identifikation des Wortinhalts behilflich sein kann" (dGWDaF 2000: X).

Alle vier Wörterbücher definieren Antonyme generell als „Wortpaare mit gegensätzlicher Bedeutung“ (WGWDaF 2008: 11), die ,auf der gleichen Bezugsebene eine Art Gegenpol zum betreffenden Stichwort" (LGWDaF 2003: XVIII) bilden. Sie werden gesondert mit Identifikationsangaben $(\leftrightarrow$, Ggs, ANT) markiert und entweder vor oder direkt nach der Bedeutungserläuterung positioniert:

(10) laut (↔ leise) so, dass etwas gut und weit hörbar ist (PGWDaF 2004: 828) laut - so, dass ihre Klänge od. Geräusche auch von weitem gehört werden können Ggs. leise (WGWDaF 2008: 637)

Dieses lexikographische Vorgehen könnte für den Lerner gewinnbringend sein, dennoch kann die im Vorwort zu allgemein formulierte Definition der Gegenwörter zur Folge haben, dass einige Vernetzungen zwischen den Stichwortbedeutungen trotz der eingesetzten Antonyme dem Lernenden unerkannt bleiben. Nur in zwei der analysierten Nachschlagewerke (LGWDaF und WGWDaF) wird der Antonymbegriff weiter gefasst, indem als Gegensätze auch Lexeme betrachtet werden, die zwar im strengen Sinne keine Antonyme sind, die aber „das Stichwort systematisch einzuordnen" (LGWDaF 2003: XVIII) helfen. Das erklärt, warum z.B. unter dem Stichwort Fahrlicht oder Halbschuh zwei Gegenwörter angegeben werden:

\section{(11) Fahrlicht $\leftrightarrow$ Standlicht, Fernlicht (LGWDaF 2003: 333) \\ (12) Halbschuh $\leftrightarrow$ Stiefel, Sandale (LGWDaF 2003: 462)}

Die Kontrastierungen dieser Art ähneln zwar der Antonymie, zwischen den angegebenen Lemmata liegt jedoch eine hyponymische Relation vor, die auf hierarchische Relationen hindeutet. In (11) und (12) handelt es sich um Kohyponyme der Hyperonyme Fahrzeugbeleuchtung und Schuh. Für den fremdsprachigen Benutzer sind solche Zuordnungen zu begrüßen, auch wenn sie keine Antonyme im engeren Sinne sind.

Im Folgenden wird anhand von stichprobeweise ausgewählten verbalen, nominalen, adjektivischen und adverbialen Stichwörtern überprüft, wie die Lemma- 
tisierungspraxis der Gegenwörter in den DaF-Wörterbüchern aussieht, d.h. welche Lexeme in den analysierten Nachschlagewerken als Antonyme fungieren und ob sich die Wörterbuchautoren an die angegebene Definition eines Antonyms halten.

Tab. 2. Antonymische Lemmata in den Lernerwörterbüchern ${ }^{7}$

\begin{tabular}{|c|c|c|c|c|c|}
\hline & Stichwort & WGWDaF & LGWDaF & PGWDaF & dGWDaF \\
\hline \multirow{20}{*}{ 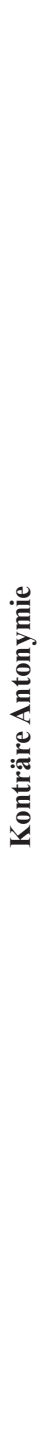 } & Licht & Ggs. Schatten & $\begin{array}{c}\leftrightarrow \text { Dunkelheit, } \\
\text { Schatten }\end{array}$ & $\leftrightarrow$ Dunkelheit & ANT Schatten \\
\hline & Schatten & Ggs Licht & - & - & - \\
\hline & Liebe & Ggs Hass & $\leftrightarrow$ Hass, & - & ANT Hass \\
\hline & Hass & Ggs Liebe & $\leftrightarrow$ Liebe & - & ANT Liebe \\
\hline & dunkel & nicht hell $\left(\mathrm{BA}^{8}\right)$ & $\leftrightarrow$ hell & - & ANT hell \\
\hline & hell & - & $\leftrightarrow$ dunkel & $\leftrightarrow$ dunkel & $\begin{array}{l}\text { ANT dunkel, } \\
\text { düster, finster }\end{array}$ \\
\hline & fröhlich & - & $\leftrightarrow$ traurig & $\leftrightarrow$ traurig & ANT traurig \\
\hline & froh & - & $\leftrightarrow$ traurig & $\leftrightarrow$ traurig & ANT traurig \\
\hline & traurig & - & $\leftrightarrow$ froh, fröhlich & $\leftrightarrow$ fröhlich & ANT fröhlich, froh \\
\hline & gesund & - & $\leftrightarrow$ krank & $\leftrightarrow$ krank & ANT krank \\
\hline & krank & $\begin{array}{c}- \\
\text { körperlich, see- } \\
\text { lisch od. geistig } \\
\text { nicht gesund (BA) }\end{array}$ & $\leftrightarrow$ gesund & $\leftrightarrow$ gesund & ANT gesund \\
\hline & gründlich & - & - & $\leftrightarrow$ oberflächlich & ANT oberflächlich \\
\hline & $\begin{array}{l}\text { oberfläch- } \\
\text { lich }\end{array}$ & $\begin{array}{l}\text { - } \\
\text { flüchtig, nicht } \\
\text { gründlich (BA) }\end{array}$ & $\begin{array}{c}- \\
\text { nicht gründlich u. } \\
\text { detailliert (BA) }\end{array}$ & - & ANT gründlich \\
\hline & nass & - & $\leftrightarrow$ trocken & $\leftrightarrow$ trocken & ANT trocken \\
\hline & feucht & - & $\leftrightarrow$ trocken & $\leftrightarrow$ trocken & ANT trocken \\
\hline & trocken & $\begin{array}{c}- \\
\text { ohne Feuchtigkeit } \\
(\mathrm{BA})\end{array}$ & $\begin{array}{l}- \\
\text { ohne Feuchtig- } \\
\text { keit, nicht nass } \\
\text { (BA) }\end{array}$ & $\leftrightarrow$ feucht & ANT nass, feucht \\
\hline & scharf & - & $\leftrightarrow$ stumpf & $\leftrightarrow$ stumpf & ANT stumpf \\
\hline & stumpf & $\begin{array}{c}- \\
\text { nicht scharf (BA) }\end{array}$ & $\leftrightarrow$ scharf & $\leftrightarrow$ scharf & ANT scharf \\
\hline & weich & - & $\leftrightarrow$ hart & - & ANT hart \\
\hline & hart & $\begin{array}{c}\text { - fest, nicht weich } \\
\text { BA }\end{array}$ & $\leftrightarrow$ weich & $\leftrightarrow$ weich & ANT weich \\
\hline
\end{tabular}

${ }^{7}$ Aus Platzgründen sind in der Tabelle (2) nur diejenigen Bedeutungsgegensätze angeführt, die in einer inhaltlichen Opposition zu der Hauptbedeutungsvariante des Lemmas stehen.

${ }^{8}$ Mit „BA“ wird markiert, dass in dem Wörterbuchartikel eine Bedeutungserläuterung angegeben ist, die aber nicht als Antonym markiert wird. 


\begin{tabular}{|c|c|c|c|c|c|}
\hline & Stichwort & WGWDaF & LGWDaF & PGWDaF & dGWDaF \\
\hline \multirow{10}{*}{ 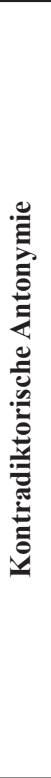 } & erkranken & - & - & - & ANT genesen \\
\hline & genesen & - & $\leftrightarrow$ erkranken & - & ANT erkrankt \\
\hline & gewinnen & - & $\leftrightarrow$ verlieren & - & ANT verlieren \\
\hline & verlieren & $\begin{array}{l}\text { - } \\
\text { ein Spiel, einen } \\
\text { Wettkampf nicht } \\
\text { gewinnen (BA) }\end{array}$ & $\leftrightarrow$ gewinnen & $\leftrightarrow$ gewinnen & ANT gewinnen \\
\hline & Hengst & Ggs Stute & $\leftrightarrow$ Stute & $\leftrightarrow$ Stute & vgl. Stute \\
\hline & Stute & Ggs Hengst & $\leftrightarrow$ Hengst & - & $\begin{array}{c} \\
\text { vgl. Hengst }\end{array}$ \\
\hline & tot & - & - & $\leftrightarrow$ lebendig & ANT lebendig \\
\hline & lebendig & - & $\leftrightarrow$ tot & $\leftrightarrow$ tot & ANT tot \\
\hline & überall & - & - & $\begin{array}{l}\leftrightarrow \text { nirgends, nir- } \\
\text { gendwo }\end{array}$ & ANT nirgends \\
\hline & $\begin{array}{l}\text { nirgend- } \\
\text { wo/ } \\
\text { nirgends }\end{array}$ & - & $\leftrightarrow$ überall & $\leftrightarrow$ überall & $\begin{array}{l}\text { ANT irgendwo } \\
\text { ANT überall }\end{array}$ \\
\hline \multirow{6}{*}{ 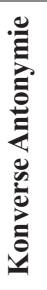 } & kaufen & - & $\leftrightarrow$ verkaufen & - & ANT verkaufen \\
\hline & verkaufen & - & $\leftrightarrow$ kaufen & - & ANT kaufen \\
\hline & links & Ggs rechts & $\leftrightarrow$ rechts & - & ANT rechts \\
\hline & rechts & Ggs links & $\leftrightarrow$ links & $\leftrightarrow$ links & ANT links \\
\hline & vorn(e) & Ggs hinten & $\leftrightarrow$ hinten & $\leftrightarrow$ hinten & ANT hinten \\
\hline & hinten & Ggs vorn & $\leftrightarrow$ vorne & $\leftrightarrow$ vorne & ANT vorn \\
\hline \multirow{8}{*}{ 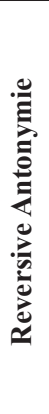 } & aufmachen & - & $\leftrightarrow$ zumachen & - & ANT zumachen \\
\hline & zumachen & - & $\leftrightarrow$ aufmachen & - & ANT aufmachen \\
\hline & beladen & - & - & - & ANT entladen \\
\hline & entladen & - & - & - & ANT beladen \\
\hline & $\begin{array}{l}\text { einschla- } \\
\text { fen }\end{array}$ & - & - & - & $\begin{array}{c}\text { ANT erwachen, } \\
\text { aufwachen }\end{array}$ \\
\hline & aufwachen & - & - & - & ANT einschlafen \\
\hline & öffnen & - & $\leftrightarrow$ schließen & $\leftrightarrow$ schließen & ANT schließen \\
\hline & schließen & - & $\leftrightarrow$ öffnen & - & ANT öffnen \\
\hline
\end{tabular}

Auf den ersten Blick ist in der Tabelle (2) zu erkennen, dass zwischen den Wörterbüchern gewisse Diskrepanzen im Hinblick auf die Begriffsbestimmung und die Markierungsweise der Antonyme festzustellen sind. Die Sichtung des Untersuchungsmaterials lässt einige Schlussfolgerungen und Überlegungen formulieren:

1. Als Antonyme werden in den analysierten Wörterbüchern in der Regel skalierbare Gegensatzwörter betrachtet, die als Gegenpole in gleichen Kontexten eingesetzt werden können, z.B. fröhlich - traurig, scharf - stumpf, nass / feucht 
- trocken, Liebe - Hass. Solche Vorgehensweise entspricht im Wesentlichen den Definitionen der Antonyme, die in den Hinweisen für den Benutzer des jeweiligen Wörterbuchs dargelegt werden. Bei Lexemen, die in komplementären (erkranken - genesen, überall - nirgendwo / nirgends) oder in konversen Relationen (kaufen - verkaufen) zueinander stehen, fehlen oft die Gegensatzangaben. Die meisten Unvollständigkeiten in der Angabe der Gegenwörter sind im Falle der reversiven Antonymie zu beobachten: Die Verben beladen - entladen, einschlafen - aufwachen werden nur im dGWDaF als Gegensatzpaare betrachtet. Bei aufmachen zumachen und öffnen - schließen steht die Information von dem entsprechenden Gegensatzverb lediglich in LGWDaF und dGWDaF.

2. Relativ häufig wird bei der Angabe des Bedeutungsgegensatzes so vorgegangen, dass das Gegenwort nur bei dem das oppositionelle Paar bildenden Lexem angegeben wird, es fehlt aber bei seinem Pendant, z.B. Schatten - Licht, (LGWDaF, PGWDaF, dGWDaF), gründlich - oberflächlich, Hengst-Stute, links - rechts, dunkel - hell (PGWDaF), tot - lebendig (LGWDaF).

3. Wie bereits erwähnt, sollen Antonyme die Unzulänglichkeiten einer sprachlichen Definition, die in der zu lernenden Sprache verfasst ist, kompensieren. Recht häufig werden aber unverständlicherweise Gegensatzwörter nicht als Antonyme markiert, obwohl sie als begleitende Lexeme in die Bedeutungserläuterung eingeschoben sind, z.B. gründlich - oberflächlich (WGWDaF, LGWDaF), gesund-krank, feucht-trocken, weich - hart, gewinnen - verlieren (WGWDaF).

4. $\mathrm{Zu}$ betonen ist, dass die Wörterbuchautoren in der Regel viel Wert auf den Nachweis der Substituierbarkeit legen. So wird die Antonymangabe auf bestimmte Kontexte eingegrenzt und mit zutreffenden Beispielen visualisiert:

(13) trocken:

1. ANT nass /auf Stoffliches bez./: trock(e)ne Kleidung, Sachen anziehen [...]

ANT feucht/ vorw. auf Körperliches bez./: trock(e)ne Haut [...], ANT saftig /auf Pflanzen, Teile von Pflanzen bez./: [...] trock(e)e Äste, Blätter

2. ANT lieblich /auf Wein, Sekt bez./ich habe uns einen trock(e)nen Wein bestellt

4. ANT interessant/ der trock(e)ne Lehrstoff liegt mir nicht (dGWDaF 2000: 1047-1048)

(14) stumpf:

1. (↔ scharf) Das Messer ist völlig stumpf.

3. (↔ teilnahmslos, abgestumpft) Er lebte nach dem Tod seiner Frau nur noch stumpf dahin. (PGWDaF 2004: 1300) 


\section{Fazit}

Wenn ein Lernerwörterbuch gleichzeitig bei Sprachrezeption, bei Sprachproduktion und als Unterrichtshilfsmittel benutzt werden soll, so muss es lexikographische Angaben nicht nur auf der syntagmatischen, sondern auch auf der paradigmatischen Ebene enthalten. Demnach sollen Similaritäts- und Kontrastrelationen in den Explikationsteil der Artikeltexte eines Lernerwörterbuchs integriert werden. Dieses lexikographische Verfahren lässt sich aus der Lernerperspektive bestens legitimieren, weil die Semantisierung über Synonymen- und Antonymenangaben eine effiziente Identifikation und Kontrastierung von Wortbedeutungen ermöglicht. Die Wörterbuchautoren sollen jedoch in den Hinweisen für den Benutzer deutlich hervorheben, dass sowohl Synonyme als auch Antonyme nur eine unterstützende Funktion bei der Semantisierung erfüllen. Sie dürfen daher nicht als lexikographischer Informationsbaustein für Textproduktion missverstanden werden.

Der Vergleich der zur Analyse stichprobeweise gewählten Lexeme lässt die bestehenden Differenzen in der Präsentationsform derselben Lemmata in den gesichteten Lernerwörterbüchern feststellen. Die Divergenzen in der Markierung sind zudem im Rahmen ein und desselben Wörterbuchs zu beobachten, was besonders bemängelt werden muss. Die Darstellungsweise der Synonyme und Antonyme in den einzelnen Wörterbüchern kann zwar je nach dem lexikographischen Konzept, je nach den favorisierten Grundsätzen der Bedeutungserläuterung und des Artikelaufbaus variieren, aber im Rahmen des jeweiligen Wörterbuchs muss auf das vorgegebene gleiche Präsentationsmuster zurückgegriffen werden.

Aus der Analyse resultiert, dass die Lernerwörterbücher verbesserungsbedürftig sind, weil sie den tatsächlichen Benutzererwartungen nicht vollständig entsprechen. In Anlehnung an die vorangegangenen Ausführungen sind einige Postulate an die Wörterbuchschreiber zu formulieren:

- klare Strukturierung von Wörterbuchartikeln,

- Anwendung einheitlicher Klassifizierungsprinzipien bei dem bestimmten Informationstyp (Synonym und Antonym),

- umfassende Markierung der Stilebene und Gebrauchsfrequenz der Lexeme,

- Einbindung der Synonyme und Antonyme in ausreichende Verwendungskontexte.

Die Qualität der DaF-Wörterbücher ist anhand des relativ geringen Untersuchungsmaterials nicht definitiv zu beurteilen, was auch nicht das Ziel der Untersuchung war. Die Ergebnisse der kontrastierenden Analyse erlauben aber einen kritischen Einblick in die Lemmatisierungspraxis in den gängigen Lernerwörterbüchern zu gewinnen und dadurch die Schwachstellen und die Unzulänglichkeiten der einzelnen Wörterbücher aufzuzeigen. 


\section{Literatur}

\section{Quellen}

dGWDaF $=$ de Gruyter. Wörterbuch Deutsch als Fremdsprache. Berlin/New York 2000.

LGWDaF $=$ Langenscheidt. Großwörterbuch Deutsch als Fremdsprache. Berlin 2003.

PGWDaF $=$ Pons. Großwörterbuch Deutsch als Fremdsprache. Stuttgart 2004.

WGWDaF = PWN/Wahrig. Großwörterbuch Deutsch als Fremdsprache. München 2008.

\section{Sekundärliteratur}

Bergenholtz, Henning (2002): Das de Gruyter Wörterbuch Deutsch als Fremdsprache und das neue Duden-Wörterbuch in zehn Bänden. Ein Vergleich im Hinblick auf die Grammatik. In: Wiegand, Herbert Ernst (Hrsg.): Perspektiven der pädagogischen Lexikographie II. Untersuchungen anhand des ,, de Gruyter Wörterbuchs Deutsch als Fremdsprache”. Tübingen. S. 35-53.

Harm, Volker (2015): Einführung in die Lexikologie. Darmstadt.

Knipf-Komlosi, Erzsebet / Rada, Roberta / Bernath, Csilla (2006): Aspekte des deutschen Wortschatzes: ausgewählte Fragen zu Wortschatz und Stil. Budapest.

Lyons, John (1980): Semantik. Bd. 1. München.

Runte, Maren (2015): Lernerlexikographie und Wortschatzerwerb. Berlin.

Schippan, Thea / Ehrhardt, Horst (2001): Lexik. In: Fleischer, Wolfgang / Helbig, Gerhard / Lerchner, Gotthard (Hrsg.): Kleine Enzyklopädie. Deutsche Sprache. Frankfurt am Main.

Töpel, Antje (2011): Der Definitionswortschatz im einsprachigen Lernerwörterbuch des Deutschen. Anspruch und Wirklichkeit. Tübingen.

Wanzeck, Christiane (2010): Lexikologie. Göttingen.

Wellmann, Hans (2004): Der Definitionswortschatz des einsprachigen Wörterbuchs. In: Herbst, Thomas et al. (Hrsg.): Lexikographie, ihre Basis- und Nachbarwissenschaften. (Englische) Wörterbücher zwischen 'common sense' und angewandter Theorie. Tübingen. S. 89-105.

Wiegand, Herbert Ernst (2013): Lexikographie und angewandte Linguistik. In: Zeitschrift für Angewandte Linguistik 58, S. 13-39.

Wiegand, Herbert Ernst et al. (2010): Wörterbuch zur Lexikographie und Wörterbuchforschung. Bd. 1. Berlin/New York.

Zöfgen, Ekkehard (1994): Lernerwörterbücher in Theorie und Praxis. Ein Beitrag zur Metalexikographie mit besonderer Berücksichtigung des Französischen. Tübingen.

\section{Abstracts}

Der vorliegende Beitrag setzt sich zum Ziel, aufgrund der kontrastierenden Analyse der gewählten Wörterbuchartikel aufzuzeigen, welche Lexeme in den gängigen deutschen Lernerwörterbüchern als Synonyme bzw. Antonyme fungieren und wie diese lemmatisiert werden. Anschließend wird der Frage nachgegangen, inwieweit die bei den paradigmatischen Austauschklassen stehenden Informationen dem potentiellen fremdsprachigen Wörterbuchbenutzer bei möglichen Fragestellungen weiterhelfen können.

Schlüsselwörter: Lernerwörterbücher, Synonymie, Antonymie, paradigmatische Relationen 


\section{Similarity and Contrast in Learner's Dictionaries}

The author carries out a contrastive analysis of some entries selected from monolingual learner's dictionaries. The analysis shows which linguistic items function as synonyms and antonyms in commonly used dictionaries and how they are lemmatized. Moreover, the corpus analysis indicates the extent to which the definitions of paradigmatically related entries may help foreign language learners to correctly choose and use appropriate lexemes.

Keywords: learner's dictionaries, synonymy, antonymy, paradigmatic relations

Hanna Kaczmarek

Akademia im. Jana Długosza w Częstochowie

Instytut Filologii Obcych

ul. Zbierskiego 2/4

42-200 Częstochowa

Polen

E-Mail: hannakaczmarek@poczta.onet.pl 